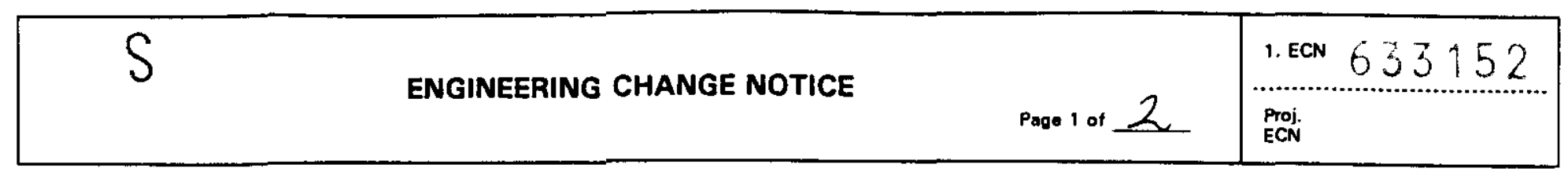

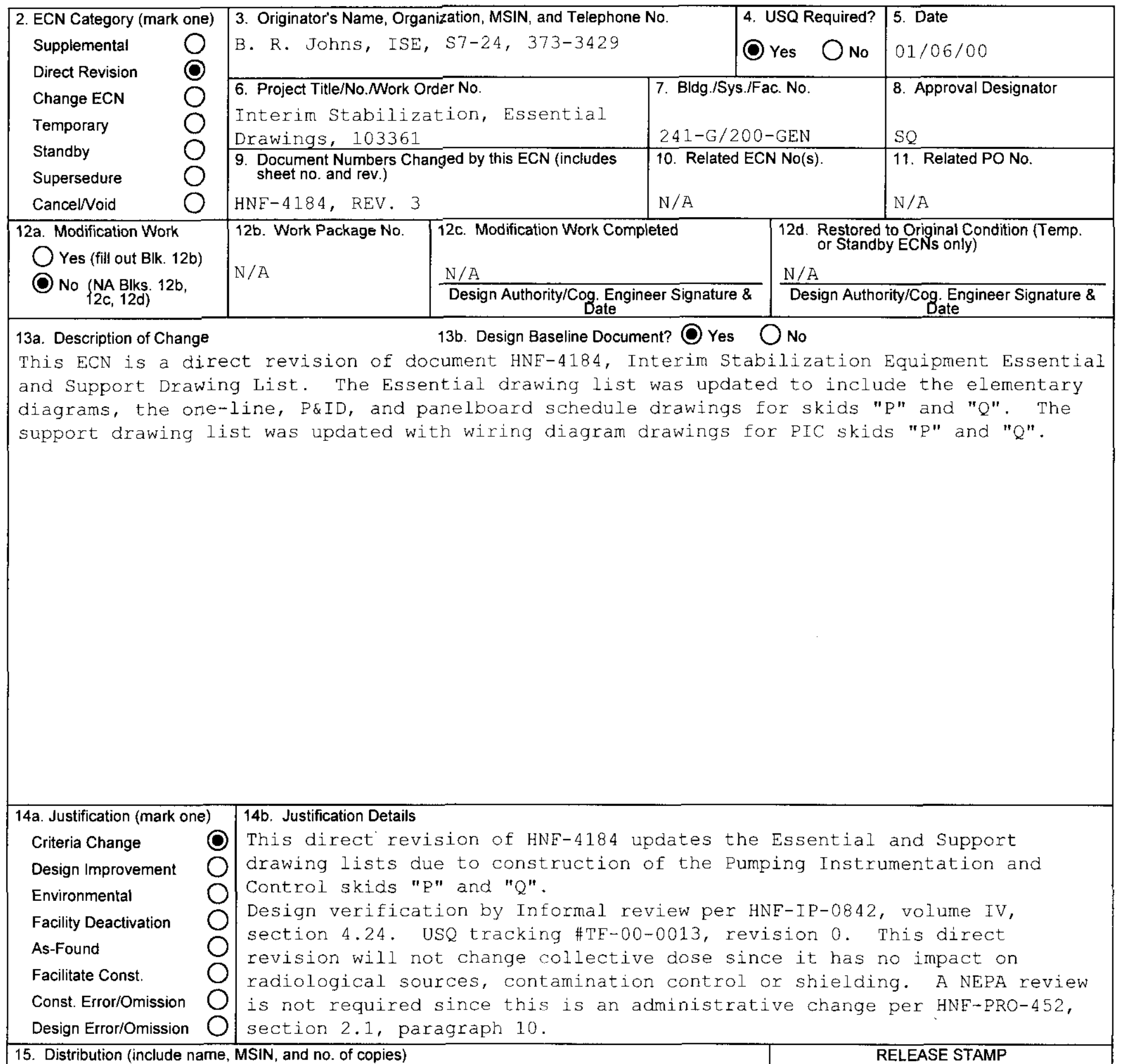

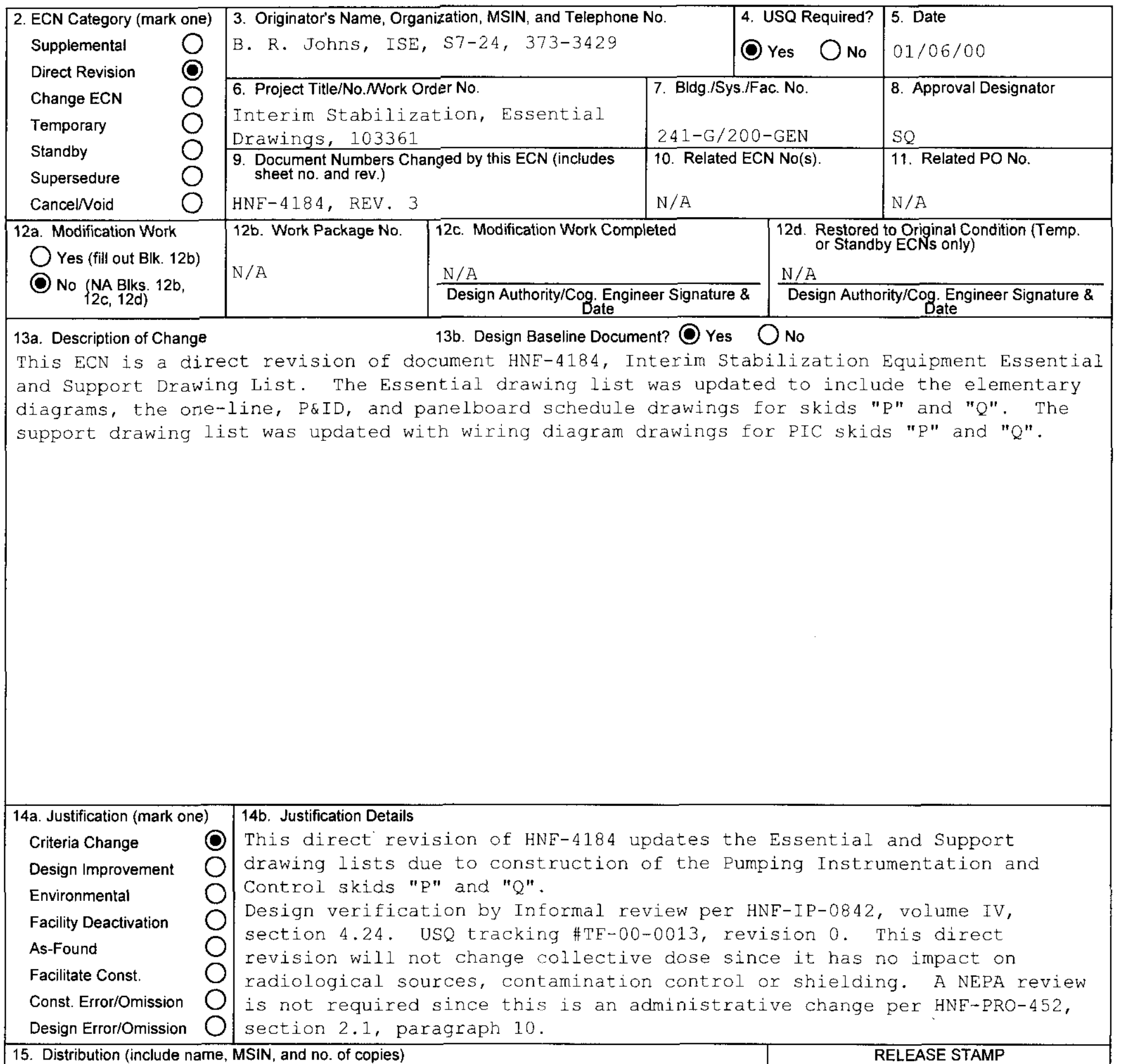

15. Distribution (include name, MSIN, and no. of copies)

O. M. Jaka $57-34$

B. R. Johns S7-24

M. R. Koch S7-24

J. E. Lamphere $57-24$

T. J. Volkman $\quad \mathrm{S} 7-34$

W. F. Zurofe S7-24

F. A. Zak $\$ 7-34$

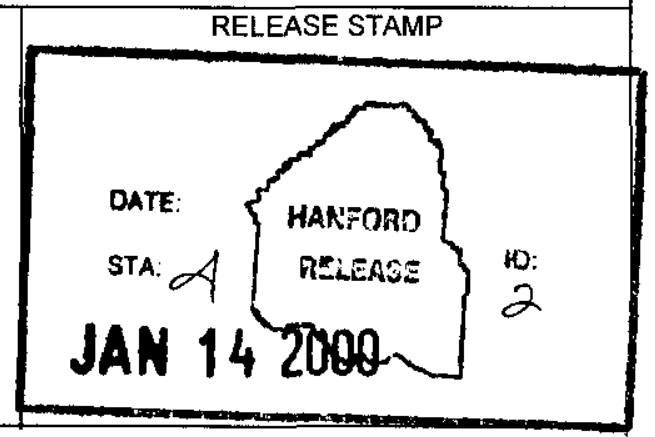




\section{ENGINEERING CHANGE NOTICE}

16. Design Verification Required

\section{O Yes \\ No}

17. Cost Impact

ENGINEERING

$\begin{array}{lll}\text { Additional } O & \$ N / A \\ \text { Savings } O & \$ N / A\end{array}$

\section{CONSTRUCTION}

Additional $\bigcirc \$ N / A$

Savings $\bigcirc \$ N / A$
Page 2 of 2

63

633152

18. Schedule Impact (days)

Improvement $\mathrm{O} \frac{\mathrm{N} / \mathrm{A}}{\mathrm{N}}$

19. Change Impact Review: Indicate the related documents (other than the engineering documents identified on Side 1) that will be affected by the change described in Block 13. Enter the affected document number in Block 20.

\section{SDD/DD}

Functional Design Criteria

Operating Specification

Criticality Specification

Conceptual Design Report

Equipment Spec.

Const. Spec.

Procurement Spec.

Vendor Information

OM Manual

FSAR/SAR

Safety Equipment List

Radiation Work Permit

Environmental Impact Statement

Environmental Report

Environmental Permit

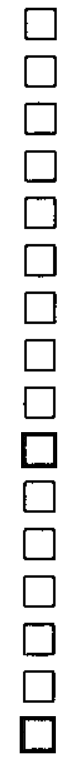

Seismic/Stress Analysis
Stress/Design Report
Interface Control Drawing
Calibration Procedure
Installation Procedure
Maintenance Procedure
Engineering Procedure
Operating Instruction
Operating Procedure
Operational Safety Requirement
IEFD Drawing
Cell Arrangement Drawing
Essential Material Specification
Fac. Proc. Samp. Schedule
Inspection Plan
Inventory Adjustment Request

Tank Calibration Manual

Health Physics Procedure

Spares Multiple Unit Listing

Test Procedures/Specification

Component Index

ASME Coded Item

Human Factor Consideration

Computer Software

Electric Circuit Schedule

ICRS Procedure

Process Control Manual/Plan

Process Flow Chart

Purchase Requisition

Tickler File

NONE

20. Other Affected Documents: (NOTE: Documents listed below will not be revised by this ECN.) Signatures below indicate that the signing organization has been notified of other affected documents listed below.

Document Number/Revision

$\mathrm{N} / \mathrm{A}$
Document Number/Revision

$\mathrm{N} / \mathrm{A}$
Document Number/Revision

21. Approvals

Design Authority WF zUROFF Cog. Eng. BR joHNs BRothen $1 / 6 / 00$ Cog. Mgr. MR KOCH Wmatris

QA

Safety

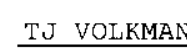

Environ. $N / A$

Other Informal Review: A $1, d x$
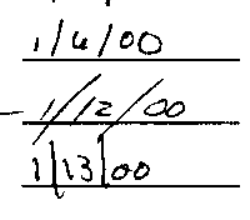

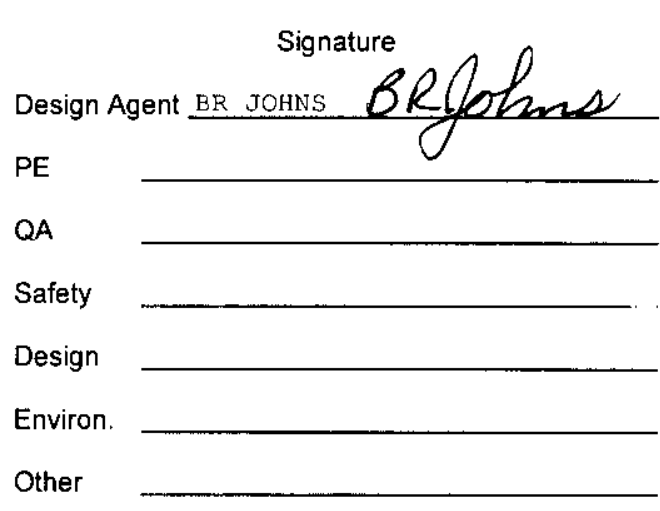

DEPARTMENT OF ENERGY

Signature or a Control Number that tracks the Approval Signature

\section{$\mathrm{N} / \mathrm{A}$}




\title{
INTERIM STABILIZATION EQUIPMENT ESSENTIAL AND SUPPORT DRAWING PLAN
}

M. R. $\mathrm{KOCH}$

CH2M HILL HANEORD GROUP, INC

Richland, WA 99352

U.S. Department of Energy Contract DE-AC06-96RL13200

\author{
EDT/ECN: 633152 UC: \\ Org Code: 74000 Charge Code: 103361 \\ B\&R Code: EW3120071 Total Pages: 8
}

Key Words: INTERIM STABILIZATION, SALTWELLS, SKIDS, DRAWINGS, PICS

Abstract:

This supporting document provides a list of the Essential and Support drawings for the Interim stabilization project equipment.

TRADEMARK DISCLAIMER. Reference herein to any specific commercial product, process, or service by trade name, trademark, manufacturer, or otherwise, does not necessarily constitute or imply its endorsement, recommendation, or favoring by the United States Government or any agency thereof or its contractors or subcontractors.

Printed in the United States of America. To obtain copies of this document, contact: Document Control Services, P.O. Box 950, Mailstop H6-08, Richland WA 99352, Phone (509) 372-2420; Fax (509) 376-4989.

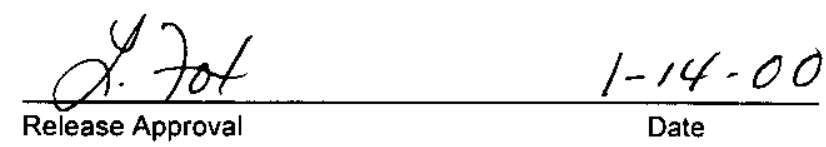

Release Approval Date

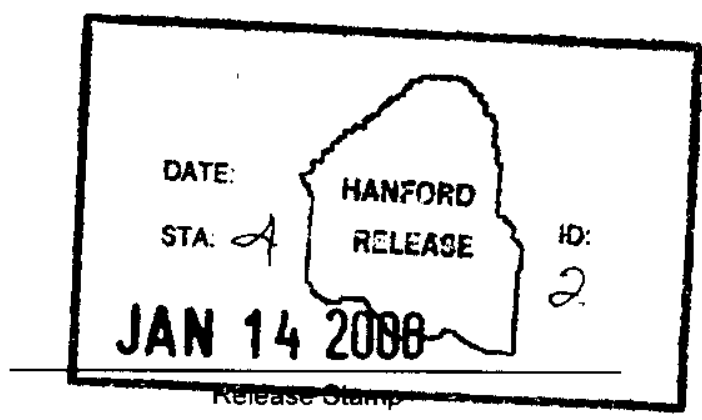

\section{Approved For Public Release}




\section{RECORD OF REVISION}

(2) Title

INTERIM STABILIZATION EQUIPMENT ESSENTIAL AND SUPPORT DRAWING PLAN

Change Control Record

(3) Revision

(7)

$0 \quad$ EDT $623362, \quad$ (ORIGINAL)

1 ECN 654142, Direct revision. Updated Essential and Support drawing lists.

ECN 648473, Direct revision. Updated Essential and Support drawing lists.

3 ECN 648491, DIRECT REVISION. Updated Essential \& Support drawings lists for skids $M$ and $N$

4

(4) Description of Change - Replace, Add, and Delete Pages

ECN 633152, DIRECT REVISION. Updated Essential \& Support drawing lists for skids $\mathrm{P}$ and $\mathrm{Q}$.
Authorized for Release

\begin{tabular}{|c|c|}
\hline (5) Cog, Engr. & (6) Cog. Mgr. \\
\hline C.B.MCVEY & M.R.KOCH $4 / 12 / 9$ \\
\hline C.B.MCVEY & M.R.KOCH $7 / 22 / 99$ \\
\hline B.R. JOHNS & $\begin{array}{l}\text { M.R. KOCH } \\
10 / 19 / 99\end{array}$ \\
\hline B.R. JOHNS & $\begin{array}{l}\text { M.R. KOCN } \\
11 / 11 / 99\end{array}$ \\
\hline BRghhns & $2 / 4$ \\
\hline
\end{tabular}

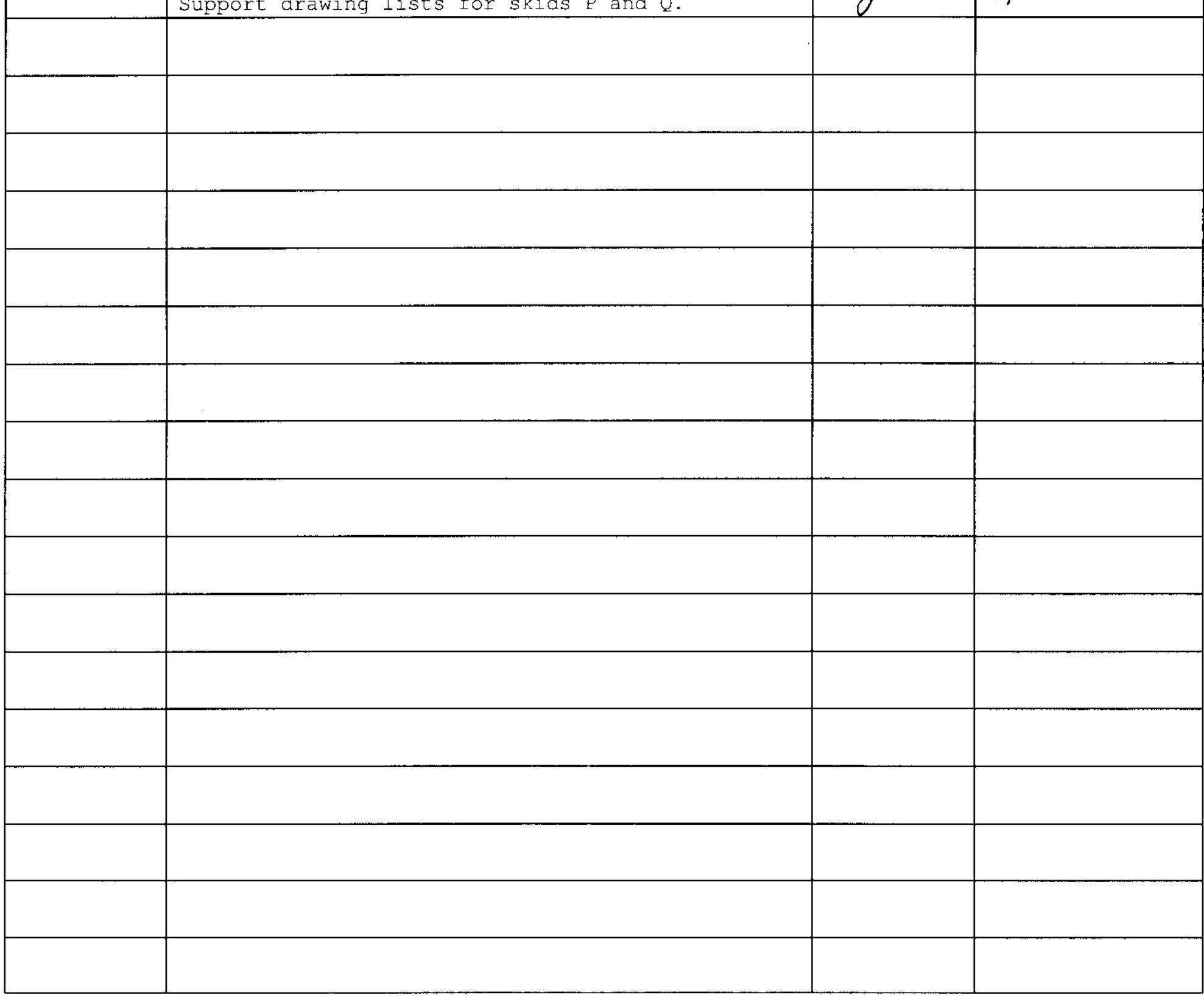


HNF-4184

REVISION 4

TABLE OF CONTENTS

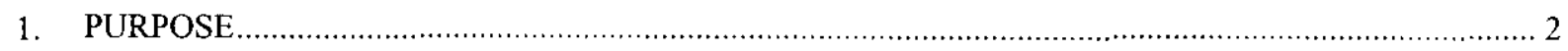

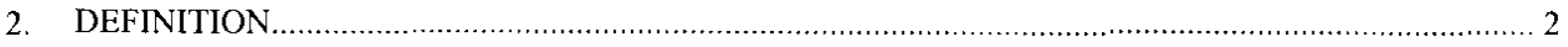

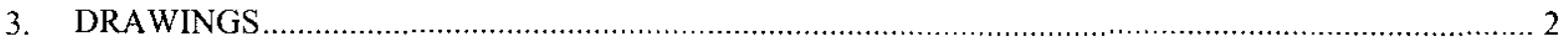

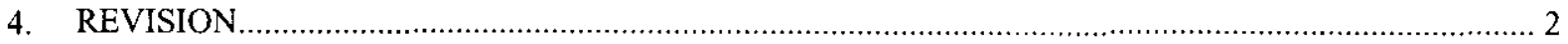

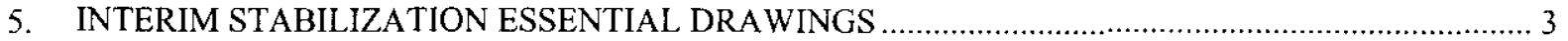

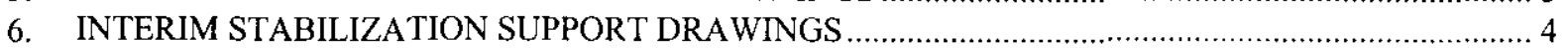

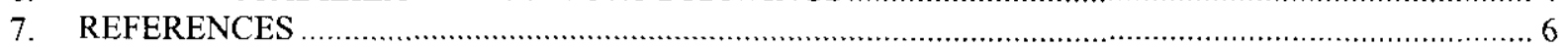




\section{PURPOSE}

The purpose of this document is to list the Interim Stabilization equipment drawings that are classified as Essential or Support drawings.

\section{DEFINITION}

Essential Drawings: Those drawings identified by the facility staff as necessary to directly support the safe operation of the facility or equipment. [HNF 1999a]

Support Drawings: Those drawings identified by the facility staff that further describe the design details of structures, systems or components shown on essential drawings. [HNF 1999a]

\section{DRAWINGS}

The Interim Stabilization drawings identified in this plan are deemed either Essential or Support drawings as defined in HNF-IP-0842, volume IV, section 4.25, paragraph 5.1.1.a, Engineering Drawings [HNF 1999a]. These drawings will be prepared, revised and

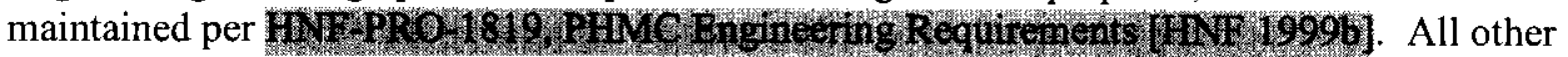
Interim Stabilization drawings not listed in this plan will be considered General drawings. Section 5 lists the Essential drawings and Section 6 lists the Support drawings.

\section{REVISION}

Drawing lists in Section 5 and Section 6 require updating when new drawings are developed or existing drawings are evaluated and determined to be Essential or Support. Drawings on the lists are to be removed when equipment functions change or equipment is removed from service. Drawings listed as Essential in this plan, but are not stamped "ESSENTIAL" shall be upgraded to Essential by an Engineering Change Notice (ECN). 
HNF-4184

REVISION 4

\section{INTERIM STABILIZATION ESSENTIAL DRAWINGS}

\section{PUMPING AND INSTRUMENTATION CONTROL SKIDS (PICS)}

\begin{tabular}{|c|c|}
\hline DRAWING NO. & DRAWING TITLE \\
\hline H-14-103530, SHT. 5 & SKID 'K' PUMPING \& INSTR CONTROL ARRANGEMENT \\
\hline H-14-103530, SHT. 13 & $\begin{array}{l}\text { SKID ‘K’ PUMPING \& INSTR CONTROL ELEMENTARY } \\
\text { DIAGRAM }\end{array}$ \\
\hline H-14-103535 & 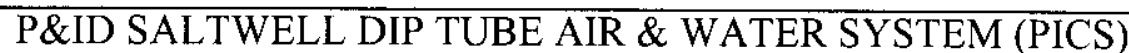 \\
\hline H-14-103536 & PANELBOARD SCHEDULE PIC SKID ‘K’ \\
\hline H-14-103537 & $\begin{array}{l}\text { PANELBOARD SCHEDULE PIC SKID 'L' } \\
\end{array}$ \\
\hline H-14-103538, SHT. 5 & SKID ‘L’ PUMPING \& INSTR CONTROL ARRANGEMENT \\
\hline H-14-103538, SHT. 13 & $\begin{array}{l}\text { SKID ‘L’ PUMPING \& INSTR CONTROL ELEMENTARY } \\
\text { DIAGRAM }\end{array}$ \\
\hline H-14-103543 & P\&ID SALTWELL DIP TUBE AIR \& WATER SYSTEM (PICS) \\
\hline H-14-103544 & PANELBOARD SCHEDULE PIC SKID 'M' \\
\hline $\mathrm{H}-14-103546$, SHT. 5 & SKID ‘M' PUMPING \& INSTR CONTROL ARRANGEMENT \\
\hline H-14-103546, SHT. 13 & $\begin{array}{l}\text { SKID ‘M' PUMPING \& INSTR CONTROL ELEMENTARY } \\
\text { DIAGRAM }\end{array}$ \\
\hline H-14-103551, SHT 1,2 & P\&ID SALTWELL DIP TUBE ȦIR \& WATER SYSTEM (PICS) \\
\hline H-14-103784, SHT. 5 & SKID “N" PUMPING \& INSTR CONTROL ARRANGEMENT \\
\hline H-14-103784, SHT. 13 & SKID "N" PUMPING \& INSTR CONTROL ARRANGEMENT \\
\hline H-14-103789, SHT. 1 & $\begin{array}{l}\text { SKID "N" P\&ID SALTWELL DIP TUBE AIR \& WATER SYS } \\
\text { (PICS) }\end{array}$ \\
\hline H-14-103790, SHT. 1 & PANELBOARD SCHEDULE PIC SKID "N" \\
\hline H-14-100791, SHT.5 & SKD"PPPUMRING \& NSTR CONTROL ARRANGEMENT \\
\hline H-141067. SII 13 & 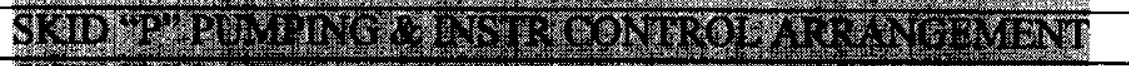 \\
\hline H1410376, sLIT & 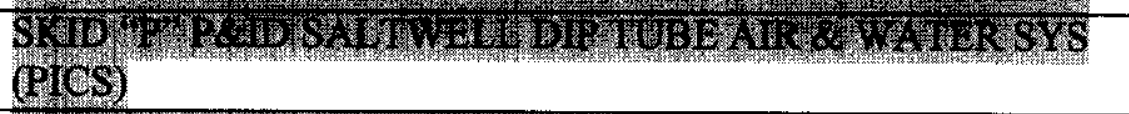 \\
\hline H-14-103797. SHA. 1 & 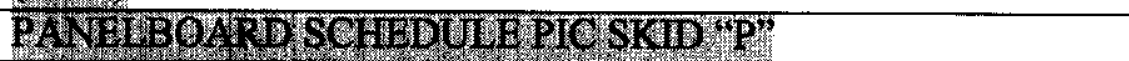 \\
\hline $11-14103798.5 H L 5$ & SKD O OWNPNG B NSIR CONTROL ARR ANGEMENT \\
\hline H14.1098\% & 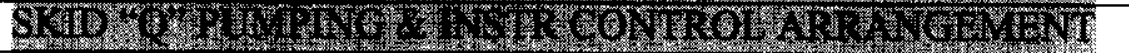 \\
\hline H-14-03810, SHT & 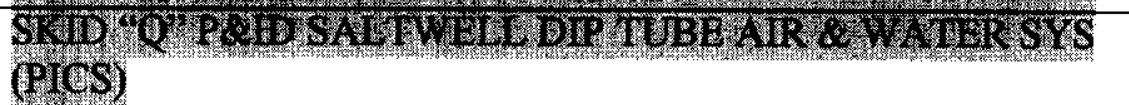 \\
\hline H414103811, SHT 1 & PANELBOARD SCHEDULE PIC SKW" $Q "$ \\
\hline & \\
\hline
\end{tabular}




\section{INTERIM STABILIZATION SUPPORT DRAWINGS}

PUMPING AND INSTRUMENTATION CONTROL SKIDS (PICS)

\begin{tabular}{|c|c|}
\hline DRAWING & \\
\hline H-14-103530, SHT 7 & SKID ‘K’ PUMPING \& INSTR CONTROL ARRANGEMENT \\
\hline H-14-103530, SHT 8 & SKID 'K' PUMPING \& INSTR CONTROL ARRANGEMENT \\
\hline H-14-103530, SHT 9 & SKID 'K' PUMPING \& INSTR CONTROL ARRANGEMENT \\
\hline H-14-103530, SHT 10 & SKID ‘K' PUMPING \& INSTR CONTROL ARRANGEMENT \\
\hline H-14-103530, SHT 11 & SKID ‘K' PUMPING \& INSTR CONTROL ARRANGEMENT \\
\hline H-14-103530, SHT 12 & SKID ‘K' PUMPING \& INSTR CONTROL ARRANGEMENT \\
\hline H-14-103533, SHT 5 & SKID 'K' LEVEL INSTR ENCL (WFIE) ARRANGEMENT \\
\hline H-14-103538, SHT 7 & SKID 'L' PUMPING \& INSTR CONTROL ARRANGEMENT \\
\hline H-14-103538, SHT 8 & SKID 'L' PUMPING \& INSTR CONTROL ARRANGEMENT \\
\hline H-14-103538, SHT 9 & SKID 'L' PUMPING \& INSTR CONTROL ARRANGEMENT \\
\hline H-14-103538, SHT 10 & SKID ‘L' PUMPING \& INSTR CONTROL ÄRRANGEMENT \\
\hline $\mathrm{H}-14-103538$, SHT 11 & SKID ‘L' PUMPING \& INSTR CONTROL ARRANGEMENT \\
\hline H-14-103538, SHT 12 & SKID 'L' PUMPING \& INSTR CONTROL ARRANGEMENT \\
\hline H-14-103541, SHT 5 & SKID ‘L' LEVEL INSTR ENCL (WFIE) ARRANGEMENT \\
\hline $\mathrm{H}-14-103546, \mathrm{SHT} 7$ & SKID 'M' PUMPING \& INSTR CONTROL ARRANGEMENT \\
\hline $\mathrm{H}-14-103546$, SHT 8 & SKID 'M'PUMPING \& INSTR CONTROL ARRANGEMENT \\
\hline H-14-103546, SHT 9 & SKID 'M' PUMPING \& INSTR CONTROL ARRANGEMENT \\
\hline H-14-103546, SHT 10 & SKID 'M' PUMPING \& INSTR CONTROL ARRANGEMENT \\
\hline $\mathrm{H}-14-103546$, SHT 11 & SKID 'M' PUMPING \& INSTR CONTROL ARRANGEMENT \\
\hline H-14-103546, SHT 12 & SKID 'M'PUMPING \& INSTR CONTROL ARRANGEMENT \\
\hline H-14-103549, SHT 5 & SKID 'M' LEVEL INSTR ENCL (WFIE) ARRANGEMENT \\
\hline $\mathrm{H}-14-103784$, SHT 7 & SKID “N" PUMPING \& INSTR CONTROL ARRĀNGEMENT \\
\hline H-14-103784, SHT 8 & SKID "N" PUMPING \& INSTR CONTROL ARRANGEMENT \\
\hline H-14-103784, SHT 9 & SKID “N" PUMPING \& INSTR CONTROL ARRANGEMENT \\
\hline H-14-103784, SHT 10 & SKID “N” PUMPING \& INSTR CONTROL ARRANGEMENT \\
\hline $\mathrm{H}-14-103784, \mathrm{SHT} 11$ & SKID “N” PUMPING \& INSTR CONTROL ARRANGEMENT \\
\hline H-14-103784, SHT 12 & SKID “N" PUMPING \& INSTR CONTROL ARRANGEMENT \\
\hline H-14-103787, SHT 5 & SKID “N” LEVEL INSTR ENCL (WFIE) ARRANGEMENT \\
\hline H14.10396. STI? & 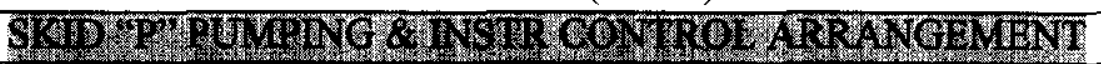 \\
\hline 114.10301, 917\%8 & 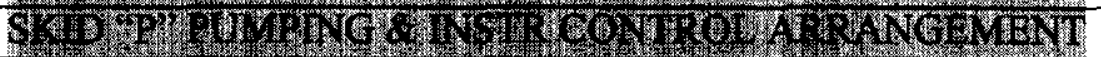 \\
\hline H.1. 10090.8149 & 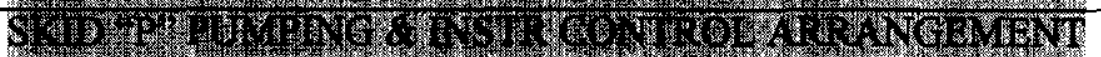 \\
\hline H14.103791, S11010 & 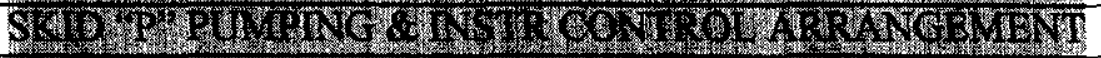 \\
\hline H. 141037918 SWII & 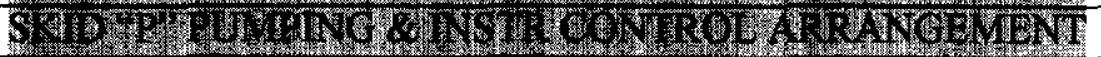 \\
\hline M14.103791 SI/12 & 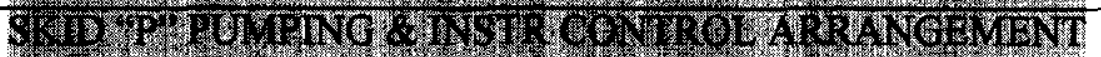 \\
\hline 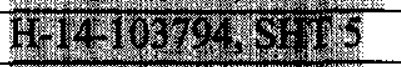 & 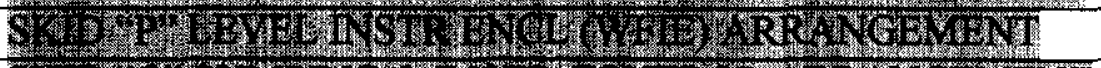 \\
\hline 91410B 98.91017 & 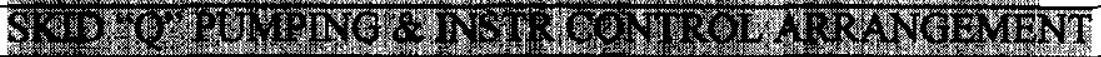 \\
\hline 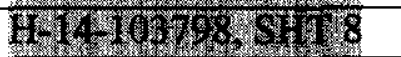 & 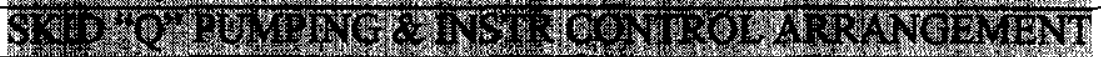 \\
\hline 114-103798, sing & 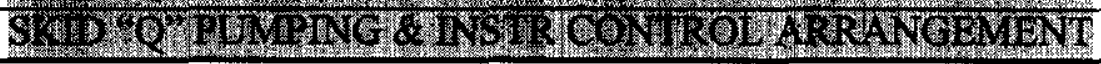 \\
\hline
\end{tabular}


HNF-4184

REVISION 4

\begin{tabular}{|c|c|}
\hline$\overline{\text { DRAWING NO. }}$ & DRAWING TITLE \\
\hline H-14-103798, SHT 10 & SKID Q PUMPIN G \& INSTR CONTROL ARRANGEMENT \\
\hline H-14 103798, SITIII & 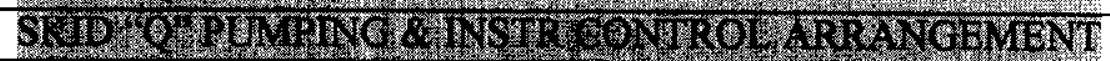 \\
\hline H-14:03798\% $/ 1 / 2$ & 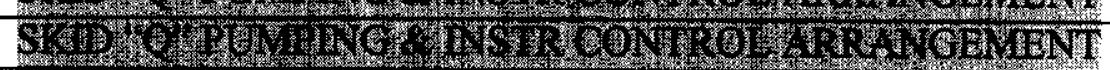 \\
\hline $1-14103801,51 T 5$ & 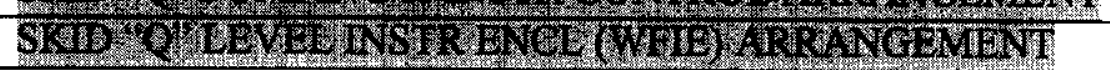 \\
\hline
\end{tabular}


HNF-4184

REVISION 4

\section{REFERENCES}

HNF 1999a, HNF-IP-0842, VOLUME IV, SECTION 4.25, REV. 0, ENGINEERING DRAWINGS, Lockheed Martin Hanford Corporation, Richland, WA.

HNF 1999b, HNF-PRO-1819, REV. 3, PHMC ENGINEERING REQUIREMENTS, Fluor Daniel Hanford, Inc, Richland, WA. 